\title{
Neural Mechanism Responses in the Brain to Predetermined Periodic and Aperiodic Stimuli - Analysis of Visual Stimulation of Movement -
}

Tadamitsu Matsuda, RPT, $\mathrm{PhD}^{1)}$, Shu Watanabe, $\mathrm{MD}, \mathrm{PhD}^{2)}$, HiRONOBU KURUMA, RPT, PhD ${ }^{2)}$, YOSHIYUKI MURAKAMI, RPT, MS ${ }^{3)}$, Rui Watanabe, RPT, MS ${ }^{4)}$, YUmi IKedA, RPT, MA ${ }^{2)}$, Atsushi Senoo, RT, PhD ${ }^{2)}$, KYOUZO YONEMOTO, MD, $\mathrm{PhD}^{2)}$

${ }^{1)}$ Department of Physical Therapy, Faculty of Health Science, Ryotokuji University: 5-8-1 Akemi, Urayasu, Chiba 279-8567, Japan. TEL: +81 47-382-2598,

E-mail: matsuda@ryotokuji-u.ac.jp

${ }^{2)}$ Graduate School of Human Health Sciences, Tokyo Metropolitan University

${ }^{3)}$ Department of Physical Therapy, Uekusa Gakuen University

4) Department of Physical Therapy, Kiyose Rehabilitation Hospital

\begin{abstract}
Purpose] Many studies have implicated the nerves connecting the basal ganglia to the supplementary motor area (SMA) in response to visual stimuli, but there have been few reports on the involvement of other areas of the brain. [Subjects] The subjects were 10 right-handed healthy adults $(8$ men, 2 women; mean age, 29.7, range: 22-47yrs) with no significant medical history or current medical problems. [Methods] We compared neural activities in the brain by functional MRI (fMRI), during a hand grasping task performed in response to periodic and aperiodic visual stimuli. [Results] Activity was significantly more extensive during performance of the aperiodic task. [Conclusion] We conclude that movement induced by unpredictable stimuli causes increased activity in the premotor cortex. Increased attention demanded by the unpredictable nature of the stimuli caused increased activation in the SMA, prefrontal cortex and cerebellum. Rehabilitation exercises paying attention to unpredictable visual stimuli may significantly influence and increase activity in the brain.
\end{abstract}

Key words: Functional magnetic imaging, Periodic-aperiodic, Motor task

(This article was submitted Dec. 8, 2009, and was accepted Jan. 12, 2010)

\section{INTRODUCTION}

The most characteristic features of walking disturbances in people with Parkinson's disease are short, quick, scuffing steps; difficulty initiating movements and sudden stopping of ongoing movement. Although the cause remains unknown, Parkinson's disease occurs due to problems in the basal ganglia as a result of dopamine deficiency in the substantia nigra.

Previous literature has suggested that Parkinson's disease is marked by deficits in timed behavior ${ }^{1,2}$. O'Reilly JX et al. ${ }^{3)}$ reported functional connectivity was enhanced between the cerebellum and the putamen in a temporal-spatial prediction task. They suggested these structures might supply the brain's metric of time.

Therefore, to investigate whether areas other than 
the basal ganglia are activated, we examined neural activities during a hand grasping task in response to either periodic or aperiodic visual stimuli using functional MRI (fMRI) in right-handed healthy subjects.

\section{SUBJECTS AND METHODS}

Ten subjects (right-handed, 8 men, 2 women; mean age, 29.7 , range: $22-47$ yrs) with no significant medical history or current medical problems were enrolled after giving their informed written consent. Handedness was determined by the Edinburgh handedness inventory ${ }^{4}$ with a lateralization index above $70 \%$. The experiment was approved by the Tokyo Metropolitan University Ethics Committee in accordance with institutional policy.

The subjects lay supine with their heads fixed to a headrest and their arms resting alongside their bodies in a 1.5 Tesla whole body MRI system and performed a repeated block pattern of tasks and rest. The blocks of two different unimanual (dominant hand) tasks were interspaced with rest times. Task blocks and rests were each of $30 \mathrm{~s}$ duration and each subject performed both tasks twice.

The visual stimuli were presented through a mirror and the subjects performed a dominant hand grasp task, while lying supine in the scanner, in response to the visual stimuli which were presented periodically $(0.5 \mathrm{~Hz})$ and aperiodically (mean frequency of $0.5 \mathrm{~Hz}$ ).

The fMRI images were captured in a 1.5 Tesla whole body MRI system equipped with an actively shielded head gradient insert and a quadrature head coil (Signa Horizon, GE). Anatomical T1-weighted images of the whole brain [multislice turbo FLASH, echo time $(\mathrm{TE})=2.4 \mathrm{~ms}$, repetition time $(\mathrm{TR})=26$ $\mathrm{ms}$, matrix $=256 \times 256$ voxels, field of view (FOV) $=24 \mathrm{~mm}$, slice thickness $=2.3 \mathrm{~mm}$, flip angle $=30^{\circ}$ ] were first obtained in the coronal, sagittal, and transverse planes to allow identification of the anterior and posterior commissures, and to determine the appropriate volume for the subsequent functional images. This volume was subsequently imaged in the transverse plane with T1-weighted echo planar imaging (EPI) $[\mathrm{TE}=90.5$ $\mathrm{ms}, \mathrm{TR}=5,000 \mathrm{~ms}$, matrix $=128 \times 128$ voxels, $\mathrm{FOV}$ $=240 \mathrm{~mm}$, slice thickness $=6 \mathrm{~mm}$, flip angle $=60^{\circ}$ ] to provide an accurate overlay of the functional images during each $180 \mathrm{~s}$ experiment, with 4 images collected in each of the task and rest periods.

The first 6 volumes of each fMRI session were discarded due to unsteady magnetization, and the remaining 36 volumes per subject were used for the analysis. The data were analyzed using statistical parametric mapping (SPM2; Wellcome Department of Cognitive Neurology, London, UK) implemented in Matlab7.0 (Mathworks, Sherborn, MA). For each subject, the functional images were realigned to correct for head movement between scans and co-registered with the subject's corresponding anatomical (T1-weighted) image. All images were normalized to a $2 \times 2 \times 2 \mathrm{~mm}^{3}$, Montreal Neurological Institute (MNI) template in Talairach space using bilinear interpolation. The anatomically normalized fMRI data were filtered using a Gaussian kernel of $8 \mathrm{~mm}$ (full width at half maximum: FWHM) in the $\mathrm{x}, \mathrm{y}$ and $\mathrm{z}$ axes.

Imaging data were modeled using a general linear model with a box-car function for block design. For the individual analysis of each motor task, one contrast was defined between the task and the baseline. Voxel-wise statistical analysis effects were first assessed in individual subjects at a voxelwise family wise error (FWE) threshold of $p<0.05$. For the individual analysis of each motor task, one contrast was defined between the task and the baseline. Voxel-wise statistical analysis effects were first assessed from the aperiodic task to periodic task comparisons of group activation at a voxel-wise FWE threshold of $p<0.05$. A statistical parametric map of the T-statistic was generated for each voxel to test hypotheses about region-specific specific condition effects.

The primary datum was the number of activated voxels calculated separately for each area of interest contralateral and ipsilateral to the hand used to perform the task. The activated foci are reported as Montreal Neurological Institute coordinates, with reference to Talairach and Tournoux. The MNI coordinates were transformed to Talairach coordinates through an established formula (http:// www.mrc-cbu.cam.ac.uk/Imaging/Common/ mnispace.shtml). The following regions of interest (ROI) were defined by WFU PickAtlas (http:// www.fmri.wfubmc.edu/cms/software) for both hemispheres: sensorimotor cortex(SMC), SMA, premotor cortex (PMC), prefrontal lobe, thalamus, occipital lobe, the basal ganglia and cerebellum of each hemisphere. 


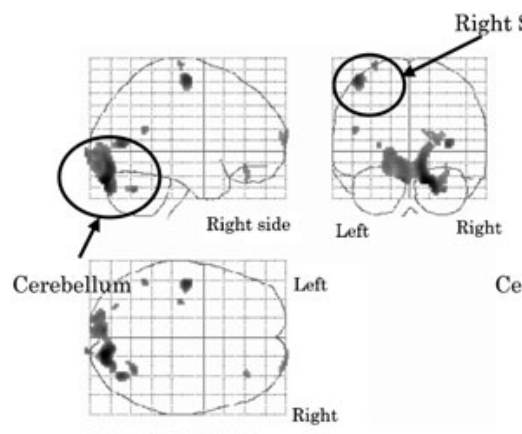

Fig. 1-1 Subjects 1

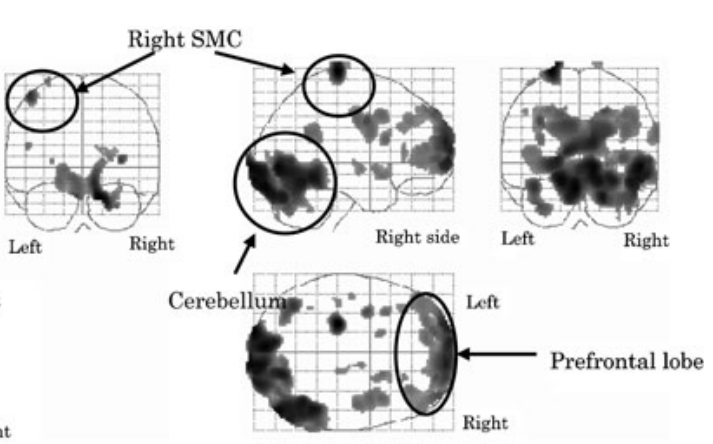

Fig. 1-2 Subjects 2

Fig. 1. Activity recorded during rest subtracted from that during the aperiodic task for 2 subjects $(\mathrm{p}<0.05 \mathrm{FWE})$.

(Views: top left, sagittal; top right, coronal; bottom left, horizontal).

\section{RESULTS}

Fig. 1-1 shows an example of activation in a 27year-old male. The subtraction of the quantity of activity that was recorded during rest from that which was recorded during performance of the aperiodic task shows activation during performance of the aperiodic task was predominantly in the left SMC, bilateral occipital lobe and cerebellum. No significant activation was observed when rest activity was subtracted from periodic activity.

Fig. 1-2 shows an example of activation in a 22year-old female. The subtraction of the quantity of activity that was recorded during rest from that which was recorded during performance of the aperiodic task shows activation during performance of the aperiodic task was predominantly in the left SMC, bilateral occipital lobe, bilateral cerebellum and prefrontal lobe. No significant activation was observed when rest activity was subtracted from periodic activity.

Fig. 2 and Table 1 show activation represented by collective data from all subjects. The subtraction of the quantity of activity that was recorded during performance of the periodic task from that which was recorded during performance of the aperiodic task showed marked activation was evident in the bilateral cerebellum vermis, bilateral caudate nucleus, right lateral dorsal nucleus, ventral anterior and lateral posterior nucleus of the thalamus, medial dorsal nucleus and VL of the bilateral thalamus, right Brodmann Areas (BA) 8, 9 and 10, and right SMA and PMC.

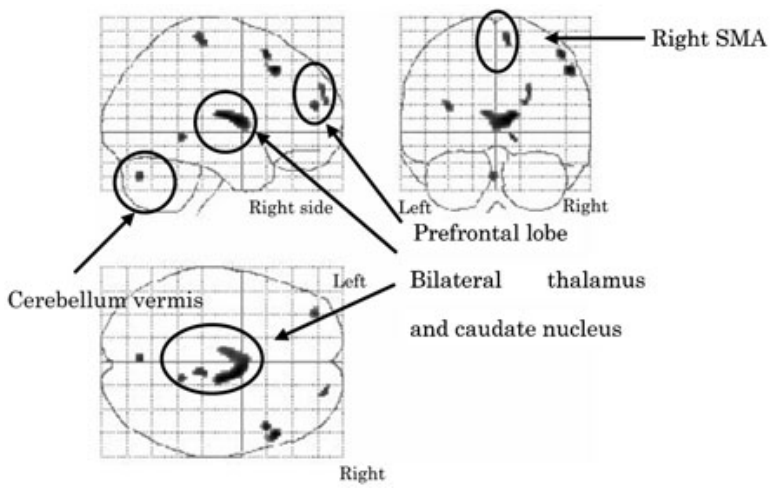

Fig. 2. Activity recorded during the periodic task subtracted from that during the aperiodic task for right-handed subjects $(n=10, p<0.05$ FWE).

(Views: top left, sagittal; top right, coronal; bottom left, horizontal).

\section{DISCUSSION}

This study of hand grasp in response to external visual, stimuli has thrown more light on the neural mechanisms of the brain (Fig. 1). Previous, studies of brain activity associated with both self-paced movements and movements in response to external cues impinging upon various sensory modalities, have reported increased activity in contralateral SMA, PMC, SMC and the cingulate motor cortex ${ }^{5)}$. Tasks with synchronized visual stimuli, were mostly associated with activation of the PMC likely reflecting preliminary associated processes. Also, it has been reported that increased activation of the right cerebellum, left SMC and SMA was higher during performance of aperiodic tasks than periodic tasks ${ }^{6)}$. Both these movement tasks involved 
Table 1. The area of brain activity and intensity for group analysis (aperiodicperiodic)

\begin{tabular}{lccrrr}
\hline & \multicolumn{2}{c}{ Right-handed subjects } & \multicolumn{3}{c}{ MNI space } \\
\hline Aperiodic - periodic & t-value & $\begin{array}{c}\text { Cluster } \\
\text { size }\end{array}$ & $x$ & $y$ & $z$ \\
\hline Right SMC & 9.91 & 524 & 58 & -20 & 48 \\
Left SMC & 10.21 & 163 & -14 & -40 & 66 \\
Right SMA & 11.97 & 680 & 8 & -24 & 64 \\
Left SMA & 11.28 & 823 & 0 & -4 & 60 \\
Right PMC & 15.03 & 398 & 6 & -28 & 66 \\
Left PMC & 11.18 & 98 & -54 & 0 & 50 \\
Right thalamus & 22.19 & 898 & 12 & -12 & 12 \\
Left thalamus & 17.24 & 672 & -6 & -8 & 12 \\
Right cerebellum & 14.01 & 163 & 16 & -42 & -28 \\
Left cerebellum & 14.45 & 200 & -2 & -72 & -30 \\
\hline Areas of brain & & & & & \\
\hline
\end{tabular}

Areas of brain showing significantly greater BOLD signal increase in the volumes of interest in right-handers in a comparison of the aperiodic and periodic tasks $(p<0.05$, FWE).

Activations are characterized by their regional maxima ( $\mathrm{t}$-value and $\mathrm{x}, \mathrm{y}, \mathrm{z}$ coordinates in MNI space). Cluster size is the number of voxels.

Abbreviations: t-value: T-statistic value.

SMC; Sensoriomotor area; SMA: supplementary motor area; PMC: premotor cortex; BA: Brodmann area.

responses synchronized with externally-triggered visual stimulus. In the present study, we found greater increase in activation of the SMA, PMC, SMC and cingulate motor cortex during unimanual performance of a visually cued aperiodic task than a visually cued periodic task. In particular, brain activation occurred more widespread throughout the premotor system during performance of the aperiodic task.

In this study, activation of the SMC was found during performance of aperiodic tasks but not periodic tasks (Fig. 1). During dominant hand grasp responses there was marked increase of activity in contralateral SMC of right-handed subjects. This finding concurs with those of a previous study on activations in the left $S_{M C}{ }^{6)}$. We found significantly greater activation of the left SMC than right during performance of aperiodic tasks.

This study may show that the reported increased activity of the cerebelleo-thalamo-cortical system during performance of an irregular finger tapping task may be attributable to additional recruitment of neurons evoked by the activity of the cerebellothalamo-cortical system ${ }^{6}$. In that study, the activated areas extended to the whole brain including the cerebrum-cerebellum. We consider that there was an increased output of motor commands in the left SMC owing to the cerebellothalamo-cortical system processing afferent and efferent information in task planning, monitoring and fine tuning during execution.

A study involving both periodic and aperiodic dominant hand index finger movements visually cued at a constant rate of $1.5 \mathrm{~Hz}$ revealed increased activation in the left precuneus, right cerebellum nucleus and vermis, left ventoral lateral nucleus of thalamus, left SMC, bilateral pre-SMA and left SMA proper ${ }^{6)}$. In contrast, movements similarly cued at the slower rate of $1 \mathrm{~Hz}$, revealed no change of activity in the $\mathrm{SMA}^{7}$ ). Also, one study of both periodic and aperiodic tasks showed greater activity in the caudal SMA than in the rostral SMA during performance of the aperiodic task ${ }^{8}$. Figure 1, and 2 show that we found greater increase of activation in the SMA and other areas, suggesting greater demand of brain activity during performance of aperiodic tasks. A comparison of brain activation for aperiodic and periodic tasks clearly shows activation of the SMA (Fig. 2). The SMA is located on the bilateral frontal medial walls and is related to planning, preparation and initiation of voluntary movements ${ }^{9-11)}$. In contrast to those findings, 
Thickbroom et al. ${ }^{8)}$ employed a slower (mean frequency of $0.5 \mathrm{~Hz}$ ) finger tapping task and found greater SMA activation for irregular than regular cueing in reactive motor operation. The discrepancy is probably because the frequency of $0.5 \mathrm{~Hz}$ allowed enough time for the subjects to predict the timing of the next movement and caused less difficulty for the regular cued task ${ }^{8}$. The results of this study support these earlier findings.

In this study, activity was greatest in the right prefrontal and premotor cortexes during performance of the aperiodic task by right-handed subjects (Fig. 1, 2). The PMC and neighboring prefrontal cortex showed increased interactions, or activity, in their neural connections than in those of the PMC and primary motor cortex. However, neural connections between the PMC and the prefrontal cortex showed more activity when subjects are thought to have monitored the next movement and are thought to have paid attention to the visual stimulus. The frontal association area is known to be related to visual and spatial attention, and the aperiodic task cued by random visual stimuli would require more visual attention to the stimulus than the periodic task which is probably more easily anticipated. It has previously been reported that the PMC modulates movement triggered or guided by external sensory cues ${ }^{12)}$. Therefore, we consider we have provided a clearer example of the neural mechanisms involved in the activated PMC.

The results show increased activity during performance of the aperiodic task in the cerebellum including the cerebellum vermis (Fig. 1, 2). In previous fMRI studies, the lateral cerebellum hemisphere and vermis showed more activity ${ }^{6,13,14)}$. The aperiodic task was not only associated with increased activation of the cerebellum, but also increased activation of both the thalamus and the motor cortex. Neural systems believed to be involved in motor control during aperiodic pacing are the cerebellar vermis and the cerebello-thalamocortical system. The correlation between neural activity in the cerebellar and thalamic nuclei and kinematic parameters was much greater when an unexpected influence led to errors during trials of a target tracking task. Also, comparable mismatches occurred during trials of an irregular tapping task and increased fMRI signals in the contralateral ventral lateral thalamic nucleus together with ipsilateral cerebellar nuclei were reported to represent error correction activity of the motor system. These findings have been reported and explanations postulated by several authors ${ }^{15,16)}$. Projections of the cerebello-thalamo-cortical system end mainly in the precentral gyrus and premotor areas.

The basal ganglia controll the whole behavior in which the frontal association area participats. The results show increased activation of areas including the basal ganglia. We consider that increased activity in relation to demands of attention involved the frontal association area. Subtraction of the quantitative activity data that was recorded during performance of the periodic task from that which was recorded during performance of the aperiodic task revealed marked activation in the caudate nucleus and the motor association loop (Fig 1, 2). In a previous study, Rao et al. reported increased activation of the left putamen and left VL during performance of a tapping task in synchrony with a constant interval auditory cue ${ }^{13)}$. In the present study, to the extent that movements cued by aperiodic visual stimuli demanded greater attention, there was increased activation of the basal ganglia.

Compared with the periodic task, activations of the brain may have been significantly more extensive during the aperiodic task for the following reasons ${ }^{6)}$ : movement induced by unpredictable outside information caused increased activity in the premotor cortex ${ }^{8)}$; increased attention demanded by the unpredictable stimulus caused increased activation in the SMA and prefrontal $\operatorname{cortex}^{7)}$; and increased activation of the cerebellum-thalamusmotor cortex because of the cerebello-thalamocortical system. We suggested that rehabilitation should be delivered mindful of the influence it has on motor tasks and brain activity after visual stimulus has been introduced ${ }^{17)}$. Aperiodic tasks need more attention. We must consider that we observed greater activity of the basal ganglia including the caudate nucleus during aperiodic task trigger movements. We will continue this line of study to see how using variations of rhythm and different movements affect brain activity.

\section{REFERENCES}

1) Pastor MA, Artieda J, Jahanshahi M, et al.: Time estimation and reproduction is abnormal in Parkinson's disease. Brain, 1992, 115: 211-215.

2) O'Boyle DJ, Freeman JS, Cody FW: The accuracy and 
precision of timing of self-paced, repetitive movements in subjects with Parkinson's disease. Brain, 1996, 119: 51-70.

3) O'Reilly JX, Mesulam MM, Nobre AC: The cerebellum predicts the timing of perceptual events. $\mathrm{J}$ Neurosci, 2008, 28: 2252-2260.

4) Oldfield RC: The assessment and analysis of handedness: the Edinburgh Inventory. Neuropsychologia, 1971, 9: 97-113.

5) Gerloff C, Richard J, Hadley J, et al.: Functional coupling and regional activation of human cortical motor areas during simple, internally paced and externally paced finger movements. Brain, 1998, 121: 1513-1531.

6) Lutz K, Specht K, Shah NJ, et al.: Tapping movements according to regular and irregular visual timing signals investigated with fMRI. Neuroreport, 2000, 11: 13011306.

7) Toma K, Ozawa M, Matsuo K, et al.: The role of the human supplementary motor area in reactive motor operation. Neurosci Lett, 2003, 344: 177-180.

8) Thickbroom GW, Byrnes ML, Sacco P, et al.: The role of the supplementary motor area in externally timed movement: the influence of predictability of movement timing. Brain Res, 2000, 874: 233-241.

9) Deecke L, Kornhuber HH: An electrical sign of participation of the mesial 'supplementary' motor cortex in human voluntary finger movement. Brain Res, 1978, 159: 473-476.
10) Tanji J, Shima K: Role for supplementary motor area cells in planning several movements ahead. Nature, 1994, 371: 413-416.

11) Thut $G$, Hauert $C$, Viviani $P$, et al.: Internally driven vs. externally cued movement selection: a study on the timing of brain activity. Brain Res Cogn Brain Res, 2000, 9: 261-269.

12) Goldberg G: Supplementary motor area structure and function: review and hypotheses. Behav Brain Sci, 1985, 8: 567-615.

13) Rao SM, Harrington DL, Haaland KY, et al.: Distributed neural systems underlying the timing of movements. J Neurosci, 1997, 17: 5528-5535.

14) Penhune VB, Zattore RJ, Evans AC: Cerebellar contributions to motor timing: a PET study of auditory and visual rhythm reproduction. J Cogn Neurosci, 1998, 10: 752-765.

15) Hore J, Villis T: A cerebellar-dependent efferents copy mechanism for generating appropriate muscle responses to limb perturbations. In: Bloedel JR(ed.), Cerebellar Functions. Berlin: Springer, 1984, pp2435.

16) Horne MK, Butler EG: The role of the cerebellothalamo-cortical pathway in skilled movement. Prog Neurobiol, 1995, 46: 199-213.

17) Kim S, Ashe J, Hendrich K, et al.: Functional magnetic resonance imaging of motor cortex: hemispheric asymmetry and handedness. Science, 1993, 261: 615617. 